\title{
ثنائية النقد والثقافة ( صراعات داخل السارد- الناقد)
}

أ.م رقية اياد احمد اعدات

كلية اللغات / جامعة بغداد المثاد

$$
\text { تاريخ الاستلام : 05-10-2020-202-16-2021 }
$$

\section{ملخص البحث}

تناول ألبحث فكرة جمع ألثقافة بالنقد واندماجها فيه حتى تصبح منهجا وأسلوبا للناقد اذ لايمكن الفصل بينهما ، ومدى تأثير الثقافة على ميول الناقد الفكرية وتحليله للنصوص ،وهي فكرة ليست بجديدة غير أن توظيفها داخل نصوص روائية عراقية هو الجديد لاسيما تأثر ألكاتب العراقي بها ، فضلا عن تأثير ظروف سياسية غير مستقرة ، واختلاف ألكتابة تبعا لما تقدم ، كما يوضح البحث أثر ألثقافة عن طريق ألناقد وفقا لثلاث محاور ، الأول :التواصلية التي يمكن أن تتحقق عن طريق النقد ، الثاني : العامية وقدرة الناقد على توظيفها وانعكاس ذلك على الثقافة العامة والخاصة ، الثالث : السارد وعلاقته بالنصوص .ان كل ماتقدم من محاور وجدت صداها في التطبيقات الخاصة بالنصوص الروائية ، لاسيما نتاجات مابعد الحرب الأمريكية على العراق ،

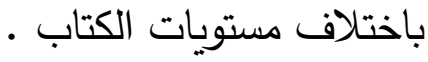

الكلمات المفتاحية: الثقافة، النقد، السارد، العامية، التواصل، التفاعلية، النصوص الروائية، الكاتب، النقد • الثقافي 


\section{Dualism of criticism and culture Conflict within the narrator- criti}

\section{Asst.lect.Ruqaya Iyad Ahmed}

\section{College of languages . University of Baghdad}

rokia_rokia@yahoo.com

$$
\text { رقم الهاتف } 07704221649
$$

Receipt date: 2020-10-05

Date of acceptance: 2021-02-16

\section{Abstract}

This research dealt with the idea of attaching culture to criticism and integrating it into it so that it becomes a method and a method for the critic. As it is not possible to separate them, and the extent of the influence of culture on the critic's intellectual inclinations and analysis of texts, as well as the influence of unstable political circumstances, and the difference in writing according to the foregoing. Based to three axes, first: the communicative that can be achieved through criticism, second: the colloquial and the ability of the critic to employ it and its reflection on the public and private culture, and the third: the narrator and itsrelationship with texts. All the foregoing themes found their resonance in the applications of fictional texts, especially the products after the US war on Iraq, at all levels of the book.

keywords: Culture , Criticism , Narrator ,Colloquial ,Communication , Interactive - Narrative texts -, Writer, and Cultural criticism 
المقدمة:

لايخفى على كل متخصص انه لايوجد أدب من غير نقد ولانقد من غير أدب وبين الاثثين يدخل الحكم الفصل ، الا وهي ثقافة الناقد بوصفها حصيلة مامر به من تجارب وخبرات وما استطاع ان يختزنه من علم مقروء ومسموع ، كل ذلك يضعه في موقف لايحسد عليه وصراع بين تقاليد واعراف مجتمعه وبين نفسه التواقة الى كل جديد ، وان كان في الجديد هذا كسرا لطوق التعاليم والقيم

مما تقدم ينطلق البحث في دراسة متواضعة لما يجول من صراعات داخل السارد الذي يصنف كناقد للمجتمع بالدرجة الاولى وهو يقدم وجهات نظر مختلفة تخضع في النهاية لسيطرته هو ، خاضعا لكل الظروف التي تعرض لها ، بل وتحداها لاسيما الحروب ومايرافقها من تضحيات اثرت بثكل سلبي على شخصية الفرد واعني في هذه الدراسة الفرد العراقي الذي انصهر بين حقبتين ، حقبة ماقبل الحرب وحقبة مابعد الحرب ، وهو يجري بين زعزعة القيم الاجتماعية وثباتها ، مرورا بزمن لامتتاه من • الخسائر

قُسِم البحث على اربعة محاور : كان الاول يدورفي فكرة من الذي بدأ اولا النقد الأدبي أم الثقافي ، وكيف نفرق بينهما ، وهل يعد قصورا اذا كان الناقد أديبا فقط ولم يكن أديبا مثقفا ، وهل في الأدب نصوص ادبية فقط وأخرى ثقافية ، كل ما تقدم من تساؤلات حاولنا الأجابة عنها في المحور الأول •

أما الثاني فيتحدث عن تواصلية النص ثقافيا ، وقدرته على الأندماج اجتماعيا وأثبات وجوده مع توالي الحقب الزمنية ، والفرق بين النص الثابت والنص المتحول . أما المحور الثالث فقد تتاول فيه الباحث العامية وتأثيرها الثقافي في السرد، واسهامها الفاعل في النهوض بالنص واستيعابه او السقوط به والنأي عن رأي النقاد ، واقتصار النص قرائيا على طبقة شعبية بعينها . أما المحور الرابع والأخير، فقد أنصب على السارد العراقي لائذا بالتقاليد الكتابية والقوانين السردية مرة ، والانفتاح الذي وفرته أجواء الاحتلال الأمريكي للعراق وظهور طبقة من مدعي الثقافة ومستهلكي النتاجات الرخيصة . 
وفي نهاية البحث قام الباحث بتثبيت النتائج والتوصيات التي من الممكن أن تتهض بالنص ثقافيا فيصبح معها صالحا للتداول . أولا : أيهما أولا النقد الأدبي أم النقد الثقافي.

عندما نتوغل في البحث في سابق أدبنا ولاحقه ، ومهما حثنا البحث في ما تفرق منه ، نجدنا عاجزين عن لملمة اشلائه ، لاسيما بعد تعرضه لعمليات السلب والحرق بسبب توالي الهجمات على المنطقة العربية ،لذلك كان حتما علينا أن نعتمد ستراتيجية بحثية خاصة نفسر بها جدلية لانهاية لها وهي :هل أن النقد وجد أولا أم النص ، ثم تتبعه جدلية أخرى هي موضوعة بحثا هل أن النقد الادبي وجد أولا أم النقد الثقافي.

مما تقدم لابد من تحديد ما اذا كان النقد وجد أولا أم النص ، والمقصود بالنص هنا هو كل ارثنا الأدبي بكل أجناسه ، واذا كان الأدب هو " حفظ اشعار العرب واخبارها والاخذ من كل علم بطرف " (ابن خلدون553، 1981)، أو "هو فن جميل يتوسل باللغة "(حسين، 148،2014)،إذن فلابد من وجود الأدب ممثلا بالنص ، ثم يتبعه النقد ، وهذا تسلسل منطقي معروف إذا ما أسقطنا من اعتبارنا أن قوانين نقدية معينة قد وضعت مسبقا كي تشكل مقاييسا لا يحيد عنها الأديب سواء كانت شكلية تتعلق بنظام القصيدة ووزنها وقافيتها على سبيل المثال ، او ضمنية كأن يجب أن تحتوي القصيدة على مقدمة ثم العرض ثم الخاتمة الخ من أنظمة استقرت عبر الزمن لنجدها أساسا واضحا ينبني عليه النص ليدخل حيز النقد ، فضلا عن أن صفات شخصية معينة تتعلق بشخص الناقد كي يتمكن من نقد أي نص يواجهه . مع ما تقدم من تصور مبدئي ، نجد أن الأدب يتقدم مثل صانعه ، ويتعقد حتى يصل إلى مرحلة الغموض والإبهام ، وكأنه شفرة تستلزم ناقدا حاذقا لفكها ، "فالادب تعبير على نحو ما عن الحدس بالاشياء والنقد على النقيض لانه الدراسة الثقافية الدقيقة لذلك التعبير ".( اندرسون 1996،10).

ولا أجدني أوافق انريك اندرسون في أن النقد على النقيض ، بل أن النص مجرد عينة أولى تفضي بالضرورة إلى نص أكبروأعمق يبتتيه الناقد على أساس النص الأول ، لذلك وحسب تقديري المتواضع كان اكان الاديب ثم تبعه النص فالثقافة أو الموهبة وثم النقد والنص هو الحد الفاصل في تقييم ثقافة هذا الأديب مها كانت ، دونية أو رفيعة ، ضعيفة أو قوية ، ويبقى النص سواء كان أدبا أم نقدا إبنا للثقافة . 
من هنا يمكننا أن نحيل أي عمل أدبي إلى شخص الناقد وشخص الكاتب معا ، إذ تدخل ردة الفعل الحسية تجاه الأشياء والحوادث والزمان والمكان ، لا بل تتعدى ذلك إلى ما يمكن أن يصيب الثخصية من أمراض نفسية معقدة تؤثر بعاقبتها على نتاج الثخص ورؤاه وإفرازاته الحسية ، لذلك كان من الاستحالة أن يتجرد الثخص ( الكاتب - الناقد ) من كل ما يشكل ثقافته ليقدم نتائج موضوعية بحتة ، ذلك أن النص صورة اخرى للحياة سواء أكان جميلا أم قبيحا ، ف" إن الاعمال الفنية .... لم تبدع لاغراض جمالية خالصة بل كان قصد منشئيها ان تتلقى على اساس ما تقوله او تمثله من معان ".(ابو زيد، 36،1994). إن ما تقدم يكثف أن لاقيامة لأدب دون نقد ، ولا نقد دون أدب ، ولا الإثنان السابقان دون ثقافة ، ولأن الرواية من مخرجات الأدب ، فقد أصبحت في الآونة الأخيرة متكأ يظهر خلالها مدى حداثة الإسلوب الروائي ومحاكاته لكل جديد ، ثم أنها تعمل كحقل نفسي تدور داخله تقلبات الأحوال البشرية سياسيا واقتصاديا واجتماعيا ونفسيا ....الخ ، أي أن عالما باكمله يطرح عن طريق الكتابة مع معالجة سوسيولوجية خاصة بالكاتب سواء كان واقعا أم متخيلا ،" أما الواقع فيتمثل في طبيعة المشكلة او التجربة اللتين يطرحهما النص ليقوم ببحثهما والناقد لايختلف عن المبدع في في اخضاع مشكلة النص او قضيته طبقا لما يسوغه النص ، لان نمط علاقة المبدع بالواقع مختلفة " (موسى، 2008ص176)، أي أن مرسل النص لابد من أن يغلف واقعه بشيء من الخيال كي يكون صالحا للتداول الفني ، ولايمكننا أن نغفل أن الكاتب يعيش صراعات داخلية تتمثل في انقسام الكاتب إلى قسمين قسم يكتب باسترسال بلا هوادة مخالفا كل ثوابته القيمية والثقافية سواء كانت دينية ام عرفية ، وقسم اخر يتحدى كل ما من شأنه ان يخرج عن ثوابته تللك ، أي ان ثنائية متصارعة تتنازع نفس الكاتب ، وقد نجده في كثير من الاحيان منقادا لكل ما يخرجه عن الطوق ويضعه في أفق حريته ، أي حريته الثخصية التي لابد أن تقوده الى حرية الفكر ، لينعكس في النهاية في اجراءاته الكتابية وإن كانت غير منطقية وخارجة عن كل ماهو مألوف ، لذلك كان جنس الرواية ومايزال وليد الأزمات ، بعد أن تكون ( الأزمات ) قد ألقت بأثقالها على الإنسان فأخرجت منه كل ما يكسر قيوده الفكرية والثقافية ، فيتجاوز بالرواية نزعة الإستمرار ، أو بمعنى أدق نزعة النمطية والإنقيادية التي تسود في "فترات الركود الإجتماعي والثقافي بوجه عام ، أو عندما تعرف قيم المجتمع نوعا من الثبات المؤسس على ما يدعم أسباب دواعي ممارسة إعادة إنتاج القيم ، ويبدأ التقطع يظهر في مراحل الرجات الهائلة التي تمس بنية المجتمع الثاملة بفعل عوامل متعددة "(يقطين 2014،ص234). 
إن أول سؤال يتبادر إلى أذهاننا فور قراءتتا للعنوان ،هو كيف يحافظ النص على ديمومته ثقافيا ؟.

لكي نحدد إجابة بعينها ، وجب علينا الدخول وبحذر إلى عالمين مختلفين تماما بفعل الزمن هو عالم الكاتب ، وعالم القاريء ، ووليدها النص ، فالنص هنا يعمل باتجاهين " باتجاه الماضي حيث يكتسب الحقيقة وباتجاه الحاضر مكتسبا المعرفة ، في هذه الأمثلة يكون للوجود المادي لنص ما .... قيمة ثقافية وتأريخية "(يقطين، 1975 :198)، تتأتى قيمته هذه من من تفاعليته مع القاريء ، بعد أن ثبت تفاعليا مع الكاتب ، بمعنى أنه انتهى كتابيا وشرع بالابتداء مع كل قراءة ، من هنا يأتي دور القاريء ثقافيا وهو يضيف ويحذف يتذكر وينسى ، يتوقف ويبتكر ، وبتصوري المتواضع أن قدرة النص ثقافيا تثبت مع النص الثعري أكثر من ثباتها مع النص السردي ( الرواية ) . شعر نقد ثقافي ثابت رواية نقد ثقافي متغير إذ أن الأول (الثعر)تتهي دلالته مع كل استحضار ، أما الثاني (الرواية)فالقاريء والناقد يتصورا مايثاءا من الصور ، مع مواصلة القراءة ، إذ أنها عاملان مع عالم باسره بصراعاته وحروبه وتقلباته ، فيصبح النص السردي متقلبا أكثر مع كل ثقافة قارئة أو ناقدة ، وهوأيضا ( النص السردي ) أكثر إفرازا للدلالة لما يحتويه من حشو للكلام والصور والوصف الذي لايغادر صغيرة ولاكبيرة ،لذلك فإن " معرفة الثقافات ووجود الجماعات والثعوب مرهون بقوة في لغاتها وما انتجته تواصليا في شكل نصوص سواء ذات طابع نفعي او فني مادامت صفة التواصل وفعاليته حادثة في تداولها "(دريدي ،مجلة فصول ،المجلد (3/25)(العدد(99)ربيع 2017م ،:30).

نبقى في دائرة ثبات النص الثعري ثقافيا ، وبتغير النص السردي في الاتجاه نفسه ، وكل مافي الأمر إن الدلالة مهما عبرت عن ثقافة زمن معين يختصرها الثاعر في كلمات معدودة ، أما النص السردي فقد ينتج الدلالة من غير توقف ، ليس بغزارة كلماته فقط ولكن بمحاكاته للمجتمع بكل جوانبه السياسية والاقتصادية والاجتماعية والقيمية والثقافية والدينية والتأريخية ، كل ما تقدم يرتبط ارتباطا جادا بتقلبات الأجواء السياسية لذلك يبقى السارد كالماشي على الحبل يتأرجح بين مايجب أن يعرضه في 
النص ، وأن المجال مفتوحا امامه بشكل حر غير مراقب ، وبين قارئه الذي يعد جلاده الأول ، كما أنه ( السارد) يواجه الحاجز الأكبر والقيد الأعظم ، ألا وهي التعاليم الدينية والقيم المتوارثة ، وهل للكاتب أو السارد أن يتحرر ومامدى حريته تلك ، وهل المستهلك له القدرة على تفه عوارضه الكتابية تلك وشفافية قلمه الذي لايمكن التخلي عنها طالما دخل عالم الكتابة عموما والسرد خصوصا ، لاسيما وهو يحرص بكل خطواته أن يتم عملية التواصل والتفاهم والترابط مع المؤلف والمستهلك ، ووفقا لما تقدم فإن الكاتب اولا واخيرا يولي عنايته بانتاجه دون البحث عن مدى التقبل او لا ، وقد نجد ذلك شاخصا في النتاجات الادبية الروائية الحديثة ، بعد ان رفعت عنها يد الرقيب نوعا ما ، لذلك فقد لانختلف اذا سلمنا "ان في الادب ايا كانت دوافعه فان إنتاج أي نص فني لزم ان يكون نصا ذاتيا بحتا يعبر عن الكاتب وافكاره أولا ، ويفرض نفسه على قارئه ثانيا وهي الحالة من الصراع بين نسق ثابت ومتحول "(عليمات، 53: 2004 :

وتبقى الثقافة بوصفها فعلا تواصليا مذبذبة بين زمنين ، زمن الكتابة وزمن التلقي ، وزمن الكاتب وزمن المتلقي ، كما إن درجات الاختلاف التي تتخلل إجراءات التواصل ، قد تصنع من النص إيقونة قابلة لإنتاج الأفكار أو مجرد قطعة فنية غير مفهومة وبلا قيمة ، لذلك كان الكاتب أو المرسل الناقد الأول لنصه ، وهو أيضا الناقد الوحيد الذي من حقه أن يفرض ثقافته على نصه ، كونه الناطق الحر المعبر عن ابناء جلدته ، لينتج نصا ليس إلا تجارب ذاتية اجتماعية ثقافية جمعية ، تكون بصمة أحداث بعينها وتحفظ تأريخا لم تتتاوله الكتب ذلك أن المرسل يجمع فيه أحداثا تتمحور وفق خيالاته وانطباعاته هو ، في الوقت ذاته يحافظ على الخيط الرفيع الذي يمكن تسميته ب( تحقيق التواصل) ، إذ لايحصل ذلك التواصل إلا بذاكرة جمعية متحققة فعليا سواء كانت قصيرة أم طويلة زمنيا " والتي تؤدي وظيفة الالية التي تتيح للثقافة حفظ نفسها والتكاثر الذاتي ، وترتكز الذاكرة الجمعية على النصوص ، فمنذ سن مبكرة تقوم بامتصاص نصوص مختلفة تشكل فضاءنا السيميوطيقي "(سمننكر ،مجلة فصول ،المجلد(3/25)،العدد 99،ربيع 2017 م :294).

إن كل ما تقدم ييرز الأعمال الأدبية التي ظهرت في العراق بشتى أنواعها لاسيما بعد سقوط بغداد والتداعيات النفسية والسياسية والاقتصادية التي رافقت السقوط ، وأبرز ما ظهر هو تأريخ النتاجات الادبية قبل السقوط وبعد السقوط ، ومن النظرة الأولى يمكن معرفة النص الأدبي ، وماإذا كان قد كتب في مرحلة ماقبل الحرب او بعدها ، وذلك يتضح في ثنايا أسلوب الكاتب وموضاعاته المطروحة للنقاش لاسيما إذا كتب وكأنه يراقب الأحداث ويلاحقها ، بل ويرتبط ذلك بحجم النتاج نفسه ، ويمتد ذلك 
التأثير إلى النقد الموجه للعمل ، ولعل ابرز مانواجهه في أعمال قبل السقوط هي غزارة في الثقافة وقلة في الإنتاج يرافقه شيء من الخوف والحذر في النقد ، لاسيما الإجتماعي والسياسي ، وفي الحقيقة أننا مهما عملنا جاهدين على توجيه النقد وحصره داخل حدود النص دون التطرق إلى ظروف ما حول النص ، لانحصد سوى نقد بارد بعيد عن أي ثقافة مزعومة إذ " لاينبغي للنقد ولايمكنه ان ينحصر في الحديث عن الكتب فهو بدوره يدلي برأيه في الحياة "(تودوروف :151:1986). وتتأصل عملية التواصل بالبحث الدؤوب عن طبقة تستطيع التعاطي مع كل ماهو مكتوب ، وقد تحقق ذلك في نتجات مابعد الحرب ، اذ اصبحت الذات المستهكة نهمة لكل ما هو يشرح مكامن الذات العراقية المتأثرة بتداعيات الأحداث السياسية المتلاحقة ، ومع الوقت والتغيرات السريعة في نواحي الحياة صارت الرواية مجرد سرد لأحداث وقعت ، لكنها تحت قيد الكاتب أسيرة لما يراه لذلك " يقودنا التطفل الذي يشير الى الراوي او مستوى نصه الى أن نستتج أن الموضوع الحقيقي للسرد هو وصف لبعض الأحداث بدلا من الأحداث نفسها وأن البطل الحقيقي هو الراوي أكثر من شخصياته "(برنس ،24:2012). إذن فإن عملية التواصل ليست بالميسورة أو الهينة قرائيا على الأقل ، لأن الأحاطة والثمول بالنص ، يلزم القاريء بالحصول على ثقافة الطبقة المجتمعية المعنية في النص ، كذلك وجب عليه أيضا " القاريء" أي يكون على دراية أولية بالكاتب وحياته وظروفه التي كونت شخصيته لاسيما الإجتماعية والثقافية ، ومن ثم فإن لا قراءة بلا ثقافة ولا ثقافة بلا تواصلية . والسؤال الذي يجب أن تتطلق منه هذه الدراسة هو ، مالذي يحدو بقاريء الرواية أو الثعر أو أي نص آخر أن يواصل قراءته ، ومالذي يفتح قريحة التواصل والتفاعل هذه ؟.

وستقدم لنا كل من الروايات الآتية مثالا على اختلاف السرد وتحولاته حسب محاور البحث المقدمة ،ورواية (كث وطن ) (شهيد 2016 )،ورواية (بوهيميا الخراب ) (صلاح2009 )، ورواية (قاريء الطين ) (فالح 2016 )، ورواية (دفاتر مفككة الاوراق ) (الهنداوي 2016). إن أول سطر يطالع القاريء في رواية ( كث وطن) هو "ابشر ياعبد الرزاق الجبران ، لقد اصبحت قوادا "(شهيد، 2016 7)،أتصور أن الكاتب استطاع وبذكاء أن يدخل القاريء إلى الرواية ويجعله متعلقا بها ، باحثا عن تقسير لهذه المقولة الصادمة ، مما يؤدي لاحقا إلى التفاعل والتواصل مع مفاصل الرواية ، وكأن الكاتب يصنع شركا ذكيا بعبارة مقتضبة ليغري القاريء بالتواصل والمتابعة ويجعله متعاطفا مع ثنايا القص وانعطافاته . 
أما عنصر الجذب والثد الذي يطرحه الكاتب في رواية ( قاريء الطين) ، فهو عرض تاريخ بغداد القديمة ومناطقها ومحلاتها وأزقتها والخوض في تفاصيل ساكنيها القدماء وعاداتهم وتقاليدهم ، وذكر بعض المواقع الأثرية القديمة لاسيما إذا ارتبطت بقصص خرافية متداولة وقصص أسطورية تحكيها الجدات ، بدأت الرواية بعبارة " أخبرته بحاجتها الماسة الى استئجار احدى غرف الخان ، إيجار غرفة في شارع السعدون سيكلفها كثيرا "(فالح 2016،9).

وقد تضعف المقدمة مخيلة القاريء ليدخل في إحباط متوال وانكفاءات لانهاية لها إلى أن تصبح الرواية بأكملها مأساة يتتقل القاريء فيها من ظلمة الى اخرى ومن انتكاسة إلى اخرى ، فلا تكاد مسحة الحزن تفارق الرواية ، فضلا عن إغراق الرواية بوصف لانهاية له ، يقول :"كان النهر شحيحا ضفافه تدفع العطر الى مسافات هائلة وبعيدة ، العطر هذا الرمز السري في الذاكرة وحينما تتسم الرائحة تتداح بعيدا جدا"(صلاح 2009 : 9 ). وللكاتب في (دفاتر مفككة الاوراق (رأي آخر في المحافظة على التواصل والتفاعل ، لاسيما الابتداء بحكمة للنساء النصيب الأعظم فيها ، يقول :"مادامت الكتابة فن التورط ، فلاجرب التورط معهن "الهنداوي ، 2016: 4 )، ولاشك أن ذكر النساء وحده يمثل عنصر جذب لايقاوم للقاريء ، فضلا عن إن الكاتب استطاع أن يثد القاريء بالإفصاح عن نيته مباشرة دون الدخول في دهاليز وأفرع ضيقة يتعدها الكاتب أحيانا للحصول على درجة إثارة معينة أو جعل تفاصيل النص مثل رموز أوأحاجي على القاريء أن يحلها كي يدخل إلى عالم الرواية ، وبمعنى أدق فالمباشرة في التصريح أحيانا تمنح النص سلاسة إسلوبية ترتكز عليه للوصول إلى حالة من الجذب المنشود . العامية وتأثيرها الثقافي في السرد

يبقى الكاتب ابن بيئته مهما استوعب من ثقافات ، ومهما تثدق بلغات وعلوم أخرى ، وتثبع بمكونات اجتماعية أثرت فيه وتأثر بها ، ذلك لأن " سيكولوجيا الفرد هي سيكولوجيا المجتمع"(للتكراس 1972 : 299 _ 300 )،الذي يدخلنا بطبيعة الحال إلى الواقع النفسي للكاتب والقاريء ، ومن ثم يحيلنا إلى متباينة نقدية ثقافية كانت نتاجا لكل ماتقدم • نجدها شاخصة في الأعمال الروائية المتقدمة ، أي في حقبة مابعد الحرب الأمريكية على العراق مثلتها الروايات سابقة الذكر ، وروايات اخرى استطاعت أن تحصد الجوائز ، إذ عالجت بموضوعاتها الكثير من ظواهر المجتمع ، وتصدت لنفس الكاتب والقاريء أكثر من التركيز على الثكل الفني ،وهنا تظهر العامية بوصفها متكاً ساندا للنص عندما تقتحمه بمفرداتها الغريبة نوعا 
ما ، وأن توظيفها الصحيح يظهر مدى ثقافة الكاتب وسعة اطلاعه وتوغله في كيفية اشتغالات العامية ووقعها النفسي على القاريء ، وقد حققت ثقافة العامة لاسيما مع النتاجات اللاحقة ما لايمكن تحقيقه على المستوى السياسي والأوساط الثقافية العالية ، لما احدثته من تقارب عاطفي واضح ، وتواصل إحداثي نجم حتما عن أن المجتمع عادة ما يشترك في الأحداث والتفاصيل والنكبات والأحزان والأفراح نفسها ، ، لذا كانت العامية بدخولها إلى مضمون النص الأدبي ليست إلا " استجابة منهجية طبيعية للتغييرات التي شهدتها عملية الإنتاج الادبي والثقافي في المجتمعات العربية الحديثة والمعاصر" (اصطيف ، مجلة فصول ، المجلد (3/25)، العدد 99، ربيع 2017:16 ).

فيما تقدم يبدو أن الكاتب يعتمد بثكل أساس على تأثير العامية وما تفرزه وسط الثريحة القارئة مهما كان تحصيلها الثقافي ، ولا بد لنا من الاعتراف أن للعامية ثقافتها وتأثيرها داخل المجتمع ، منها التقرب بتؤدة إلى الميول النفسية للقاريء ، وكذلك سرعة الإستجابة الواضحة عند قراءة النص ، بما توفره من تواصل اجتماعي لاسيما في عرض أحداث لايفهها سوى الكاتب والقاريء كونهما ينتميان إلى المتمع نفسه ، هذا إذا أدركنا أن القراءة لاتتفك ترتبط "بالتنظيم الاجتماعي للآلة الكتابية ، ولكن ايضا بالقيم المستثمرة في فعل القراءة وفي الأوضاع النفسية - السوسيولوجية للافراد والمجتمع " (لينهارد ، مجلة الفكر العربي المعاصر ، العدد 13، 1981 :1481)،وهنا تكون العامية بيد الكاتب سلاحا ذا حدين ، ويمكننا أن نحصي شيئا من مميزات العامية مع شيء من مساوئها في ما يأتي من نقاط : 1- توفر العامية قاعدة عاطفية للنص على المستوى القرائي الشعبي • 2- ألترويج السريع للعمل الأدبي لما تحققه من تفاعل . 3- ألتعريف ببعض المفردات المتداولة لاسيما للمجتمعات العربية الأخرى . 4- ألاتكاء على العامية في نقل المحتوى العاطفي يؤدي إلى هجرة الفصحى شيئا فثيئا . 5- 5جرة النقاد العمل لبعده عن الإفهام • - 5 - 5

6- ألابتعاد عن العربية والعالمية ، بمعنى أدق الابتعاد عن التواصل العربي والعالمي والاقتراب بسذاجة إلى لغة الثارع . 
وإذا كانت الثقافة"تثمل كل الانثطة والاهتمامات المميزة لثعب ما "(كوبر ،2008م : 20 )،فان مهمة الكاتب لاتخلو من صعوبة كونه حلقة الوصل بين ماهو اجتماعي ثقافي شعبي ، وبين ماهو ثقافي عالمي ، مما يجعل من عملية النقد اللاحقة عملية توضيح وتحليل وإرساء لمجمعة جديدة من الأدوات الأسلوبية المتمثلة باشتغالات العامية في النص الأدبي ، إذ يبدأ النقد ب" التذوق أي القدرة على التمييز ويعبر منها الى التقسير والتعليل والتحليل والتتييم خطوات لا تغني احداهما عن الاخرى (5) : (5باس 1983م) ولابد لنا من الاعتراف بأن النتاجات الأدبية العراقية بالذات ، اتسمت بغزارتها لاسيما بعد الاحتلال الأمريكي ، في الوقت ذاته ابتعدت أغلب الأعمال عن التميز والإبداع ، وهذا ما جعل فن الكتابة عرضة لكل من هب ودب ، إذ أن الكثير من النتاجات المطروحة ليست سوى مذكرات أو تداعيات ، تعبر عن مزاجيات أصحابها وتدل على قلة خبرة بالكتابة ، والجهل بأدواتها ، لذلك برزت العامية بقوة بوصفها أسهل تعبيرا وأوسع استخداما في الكتابات الجديدة أو بمعنى أدق الطارئة . وإذا كانت العامية بطريقة ما هي تعبير عن الهوية ، فسوف نستطيع القول أنه لايمكن للهوية أن تبرز بفنيتها وجماليتها إلا بالتعاطي مع الآخر والاندماج معه وهذا يفسر لنا إصرار بعض الكتاب على استخدام مفردات شعبية أو عامية لاسيما وهم يعيشون خارج أوطانهم ، وبهذا يجابهون خطر اضمحلال هويتهم أو زوالها ، ذلك أن أخطر ما يجابه الهوية الفردية " هو المجتمع الحاشد ممثلا بمؤسسات بيروقراطية روتينية تثعر الأفراد بفقد الإحساس بالهوية الثخصية فتتداولهم بسهولة شديدة (212: (ايزايرجر 2003م)" ولعل المغالاة باستخدام العامية تؤدي إلى : 1-ألابتعاد عن الفصحى أو فالنقل القاسم المشترك للهوية العربية . 2-ألابتعاد عن الوسط العربي والعالمي معا ، أو بمعنى أدق التواصل العربي والثقافي بالاقتراب بسذاجة واضحة من ثقافة الشارع 3-تحقيق قاعدة عاطفية للنص ، ومن ثم الترويج السريع للعمل الأدبي داخليا . 4-ألتعريف ببعض المفردات العامية المتداولة لاسيما للثقافات العربية . 
5-عادة ما تودي العامية بحياة النص بسبب هجرة النقاد له لضعف ثقافتهم بلهجة الكاتب أي بالعامية التي يستخدمها ـ

وفي النهاية فان الكاتب مخير في أن يقدم نصا مع ألفاظ عامية تواصلية الهدف منها مس عاطفة القاريء واستحضار ما خفي من تداعيات ثقافية قرائية كانت أم نقدية ، ليكون القاريء خلفيته النصية الخاصة به ، والمستتبطة أو المثارة جراء استخدام العامية ، والمقصود بالخلفية النصية هي "مجموع القيم البنيوية التي تتكون منها مجموعة من النصوص ، والتي بفعل التربية وعناصر التحكم الثقافي والفني (مؤسسات ، نقد....) في بيئة سوسيو - تأريخية معينة تأخذ فيها طابع الهيمنة ، إنتاجا وتلقيا وتصبح هي مؤشر تفاعلنا وانفعالنا، (يقطين ، :952014).

في ( قاريء الطين) نقرأ " فصاح بهم حجي عباس بلهجته الجنوبية ، وصوته المبحوح " جا بس فصمات محمد صارن نخلات ن وعتقن ركبة سلمان .... شمالكم مابيكم حظ ياهو اليحجيلكم حجايتين تصدكون بي "....هسة انت شمالك مصدك بروحك مدير شركة باتا للجلود بالعراق ، سكرت حميد شبانة ؟ ضحك الجميع على سكرة نشوتهم ونام ستار بينما كانت الابتسامة مرسومة على وجهاه"فالح ، 2016، 25، في هذه الرواية تثتغل العامية مثل محرك يربط ويحرك ما تثتت من اطراف الرواية ، فهي لسان حال الثارع تعبر عن تصورهم البسيط وتبرز بوصفها صورة واضحة لطريقة تفكيرهم فجاء التوظيف في مكانه الصحيح ، كما ان العامية تظهر مدى ثقافة الروائي اوالكاتب بادق تفاصيل مجتمعه ، والنقطة الاهم ان العامية عندما تتخلل اجزاء الرواية تظهر براعة تفكير الطبقات الثعبية على بساطتها ، والذكاء و القدرة على التحليل والحوارليس حكرا على الطبقات المثققة ، لاسيما أن العامية وباللهجة الجنوبية لها وقعا خاصا يتناغم ويتفاعل في التعبير عن الفكرة وتقربها وتحبيبها إلى القاريء . أما في رواية (كث وطن) فقد تختلف وجهة نظر الكاتب في رؤيته للعامية إذ صدرها روايته فصارت عنوانا لها "كث" وهي لفظة عامية يستخدمها أهل بغداد لطرد الحيوانات ، ولأن الرواية مأساوية بطبيعتها ، إذ تتكلم عن أسير عراقي تم أسره في الحرب ، ومع كل ويلات الأسر وماتحمله من الآلام، وهو يحلم بالرجوع الى الوطن الذي لم يحمل عنه سوى الصور والذكريات الجميلة التي تركها خلفه ، وعندما تم له ذلك الرجوع لم يجد سوى الخراب والبطالة والفقر ، فقرر أن يعمل "قوادا " ، وقد أفصح الكاتب عن طبيعة الرواية وارتباط العنوان بالابتذال ، ففي الصفحة الخامسة أي بداية الرواية نجد العبارة " قبل اعدامه كانت وصيته 
الاخيرة : رجاء لاترفعوا النقاط الثلاث "(شهيد ، 2016م : 5)، ولاشك أن الثقافة العامية تحديدا وحدها التي تستطيع تمييز ما مر آنفا من عنوان الرواية أولا ، ثم ارتباطه بعبارة إفتتح بها روايته .

ولفوزي الهنداوي توظيف أصلح به من شأن عامية الثارع ، فاصبح يستخدم مايدعى باللغة الوسط ،فالقاريء العراقي يستثعرها من سياق الكلام ، ويفهم مقاصدها وتأويلاتها وإن لم يصرح بها الكاتب ، ومن أبرز مظاهر العامية في السياق أن الجملة فيها لاتفتقد لأدوات الربط وجودة السبك ، غير أنها تمتاز بقدرتها على الإفهام للقاريء العراقي تحديدا والقاريء العربي

مما تقدم تظهر ثقافة الكاتب واشتغالاتها على النصوص الروائية وتباينها متمثلة بالغروق الفردية الثقافية ، فمنهم من هو أستاذ جامعي له خبرته في تطويع اللغة ، ومنهم من هو شخص بسيط الثقافة انعكست ثقافته في نصوصه الروائية ، لاسيما إن تباين الثقافة أصلا يمكن ملاحظته في نتاجات ماقبل الحرب ونتاجات مابعد الحرب كما أسلفنا . 4-السارد العراقي

يمكننا أن نعرف الثقافة المطروحة عبر الأدب بأنها :مجموعة من انجازات الابداع الانساني ، بل كل مااضافه الانسان للطبيعة، لنتوصل إلى فهم نتاج السارد العراقي بين حقبتين ، حقبة ماقبل سقوط بغداد وحقبة ما بعد السقوط ، إذ تتثأ ثنائية ضدية مابين هذه وتلك ، بين الإلتزام والتزمت ، وبين الإفلات والانفلات ، لا بل أصبح الاختلاف أكثر وضوحا لاسيما في الموضوعات التي تتاولها الأدب ، ذلك أن الخوف من السلطة والمطاردات الاستخباراتية إنعكس في تتاول موضوعات مستهلكة متداولة ، مثل قصص عن حياة العراق أيام الاحتلال أو روايات عن علاقات غرامية فاشلة ، أو تتاول أحداث جرت أيام الحكم الملكي والثورة وتغيير الحكم ....الخ ، كل ماسبق من موضوعات كانت خاضعة لرقابة صارمة تفرضها جهات مرتبطة بالحكومة ، غير أننا لانستطيع أن ننكر أن بعض النصوص إنمازت بفنية وحرفية عالية ، لكنها تبقى حبيسة الموضوع ، رهينة لثقافة مفروضة سياسية كانت أم اجتماعية مما يقيد حرية الكتابة والاسترسال في السرد ، كما يجعل من الكاتب أسيرا لموضوعات محددة مسبقا ، لهذا اقتصرت الكتابة على أسماء بعينها كانت قد لمعت سياسيا أيضا ، أو لها علاقات سياسية تحميها على أقل تقدير · وتأسيسا على ما سبق من أن معظم النتاجات كانت تتمتع بفنية عالية خلافا لما نراه من نتاجات اليوم ، فنستطيع أن نقول إن الأدب وهو يمثل كل نواحي الحياة ، طاله التراخي والخروج عن النمطية مثله مثل باقي نواحي الحياة الأخرى ، لاسيما السياسية 
والاقتصادية والاجتماعية ...الخ، وبتصور بسيط أرى أن الأدب نال حضه الأوفر من الانفلات عن القيود القديمة والانفتاح على كل ما هو جديد ، مما أنتج كتابا ليسوا أكثر من هواة ونصوصا ليست إلا خواطر أو انثيالات أو تداعيات متأثرة بالواقع وتحولاته ولأن الأدب بحد ذاته هو انفتاح على المتمع ، فإن هدفه الأول هو التركيز على " الجماهير الايجابية الفعالية أو القراءات المقاومة والنصوص المعارضة واللحظات الطوباوية وما إلى ذلك يصف حقبة يتم فيها تدريب الأفراد على أن يكونوا مستهلكي ميديا أكثر إيجابية ، حيث تقدم لهم خيارات أوسع بكثير من المواد الثقافية "(كلنر ، مجلة فصول ، المجلد ( 3/25) العدد 99 ، ربيع 2017م:249).

إن ماتقدم يؤثر بشكل أو بآخر في طريقة استهلاك النص ، مثلما يؤثر في البدء في طريقة كتابته ، بمعنى آخر فإن قراءات ما بعد الحرب ( الحرب الامريكية على العراق ) ،قراءات تتمتع بالحرية الكاملة كما تمتازبالسعة والانفتاح على النتاج العالمي ، وإن كان لها جوانبها السلبية ، وهي أشبه ب"حالة من الصراع بين نسق ثابت ونسق ثابت ومتحول "(عليمات،2004م : 53)، مما أسس لشخصية الصعلوك ، وشخصية الصعلوك هذه تنعكس على الكاتب والمستهلك ، إذ نشأت ثقافة جديدة هي بين ملتزم بثوابت الكتابة وأصولها وبين الانفلات من حدودها تحت مسمى ( الحداثة ) ، مثل ذلك نجده عند المستهلك ، فنراه يتأرجح بين نصوص ضعيفة قد توضع في مصافي الأعمال الناجحة على الرغم من ضعفها إذا ما وضعت في أطر ومقاييس الرصانة الأدبية ، ومرد ذلك إلى إقبال القاريء الذي يعد في هذه الحال قارئا جاهلا أو ضعيفا ، لاسيما قاريء ما بعد الحرب ، مما يؤثر بدوره على خلق هوة سحيقة بين قاريء محافظ يتميز بثقافة واسعة وبين قاريء لايرجو من قراءته إلا التسلية أو الاطلاع ، بعيدا عن عين الناقد المثقف . ففي كل رواية من الروايات آنفة الذكر نجد أن السارد يدخل إلى نصه حسب ثقافته الخاصة وحسب رؤية أوجدها لنفسه ليضع لنفسه مكانا خاصا في الرواية ، يعبر عنه أما بسرده لمواقف تأريخية سابقة كما في ( قاريء الطين ، أو يحيط نفسه بجو قاتم حالك عن طريق تصوير الزوايا المظلمة للمكان كما في ( بوهيميا الخراب ) ، أو يصنع لنفسه حياة خاصة يملؤها الثذوذ والنفور إلى أبعد الحدود مع الكثير من التتاقضات كما في ( كث وطن ) ، أو يتسلل بخطى وئيدة وباستعراض مبدئي لمواقف ثقافية جمعت شخصياته كما في (دفاتر مفككة الاوراق ) · 
نقرأ في قاريء الطين " أنت تبحث عن يهودي كانت له صلة بخضوري ـ نعم يعرفه وأنت تعرفين أكثر من ذلك بدليل معرفتك

$$
\text { عن مذكراته ومكانها"(فالح ،2016م : 166). }
$$

إن جمع الحوادث التاريخية وربطها بالحوادث اللاحقة والتعاطف معها جعل الكاتب يتشتت وسط روايته وهو يحلل ويرتب ظهور شخصياته ، وفق رؤيته الخاصة طبعا ، فضلا عن أن حشد الثخصيات جعل الكاتب يتحول إلى مجرد قاص يلاحق الأحداث دون رعاية أسلوبية واضحة ، أو إطار فني يخرج الرواية من نطاق الملل ( ملل المتابعة ) ، ولعل ذلك راجع إلى أن الكاتب لايزال في بداياته وهذا أبرز ما يلاحظ على نتاجات مابعد الحرب .

أما في دفاتر مفككة الأوراق فالكاتب حاذق في سرد أحداثه ولملمة أطراف الرواية ، لابل تمكن من صنع روايات ثلاث داخل رواية واحدة ، فقد غلبت على سرده صيغة فنية لاتخلو من سخرية في بعض مفاصلها ، مما يفصح عن تمكن واضح من أدواته ، يقول " جلبت الدفاتر الثلاث إلى البيت ، تفرغت لقراءتها ..... وجدت في الدفاتر مايصلح لكتابة مادة روائية بعد اعادة إنتاجها " (الهنداوي ،2016م :67 )، ولعل هذا التمكن يتأتى من خبرة ومتابعة وقراءة وثقافة عامة أفرزتها معاصرة أحداث ماقبل الحرب ومابعدها بكل جوانبها السياسية والاجتماعية والاقتصادية .....الخ ، وإن أبرز ما يميز الكاتب العراقي عن غيره هو اندماجه بظروفه لحد الانصهار فلايستطيع الانفكاك من تأثيرها ، لذلك اصبحت أغلب النتاجات هي انعكاسات وانثيالات عاطفية تحكم تحركات السارد اثثاء عملية السرد ، فيرزح تحت وطأة التقاليد تارة على الرغم من الانفكاك عنها ظاهريا ،وتارة ينقاد لتأثراته الثخصية ، ففي روايتي (بوهيميا الخراب وكش وطن ) يعرض السارد لطبقات المجتمع الضحلة والثاذة ، ولايتردد في وصف أدق تفاصيل هذه الحياة ، بغية كسب عطف القاريء وشد انتباهه لمثل هذه الطبقات المنسية والمطحونة من ( بغايا وفقراء وأسرى عائدون من الحرب بلا مستقبل ومواطن مطارد سياسيا ومهدد بالاعدام ، وموظفون تحت خط الفقر وسكارى متسكعون بلا مأوى ولا مصدر عيش ) مثل ما تقدم من شخصيات أصبح وجودها رمزا لكتابات ما بعد الحرب ، وهذ لايعني أنها غير موجودة قبل الحرب ، غير أن التعرض لها لم يكن مباحا مثلما هي عليه الآن • مما سبق نستطيع أن نقول أن الرواية العراقية ولدت من رحم الحرب ونتائجها ،وموضوعاتها الواقعية جاءت بعد الحرب وليس قبلها (حرب سقوط بغداد) ،لذا مازال الكاتب العراقي يتهجس خطواته الأولى ، لكن بجرأة غير معهودة فيه . 
1- النقد الأدبي هو نفسه النقد الثقافي ، وليس سابقا له او لاحقا به ، ذلك أن النقد ما هو إلا وجه آخر للثقافة .

2- إن النص يتقدم ويستحدث مثل صانعه وناقده وثقافة متلقيه حتى يصل إلى مرحلة الاستغلاق والإبهام ، لذلك صار حتما أن تلحق به ثقافة ناقده فتتاوله بثكل مغاير لما تتاوله سابقيه من النقاد.

3- يعيش الكاتب أثناء كتابة النص صراعا نفسيا يقسمه على قسمين ، قسم مدفوع بلا هوادة ودون مراعاة للقيم والتقاليد

الاجتماعية والأدبية ، وقسم متردد مقيد بكل ما سبق ، ولعل النتاجات الحديثة قد تخطت حاجز الخوف .

4- الحفاظ على تواصلية النص هو الهم الأول للكاتب ، والناقد والقاريء ، يثبتان ذلك ، أو ينفيانه ، مهما كانت لغة النص

منحدرة وهي تتزل إلى لغة الثارع ، أو منتقاة بحذر ثقافي متتاه .

5- إختلفت نتاجات ما بعد الحر ب( سقوط بغداد) عن ماقبل الحرب ، في الكم والنوع والحرفية والهواية ، والتواصل

والإنقطاع ، مما يعني أن الكاتب يمكن أن ينقسم على ثلاثة اقسام : كلاسيكي ، ومجدد ، وقسم لافضل له سوى سرد الاحداث .

6- خرجت رواية ما بعد الحرب محملة بالمزيد من القصص التي ضاعت ، اغلبها في ثنايا السرد ، ولأن كتابها طارئون

على ساحة الأدب فالكثير من النتاجات سقطت في هاوية القص المباشر فأصبح كتابها قصاصين لاأكثر .

المصادر

1- مقدمة ابن خلدون ،عبد الرحمن ابن خلدون ، دار القلم ، بيروت ،ط1981،4.

2-حديث الاربعاء ،طه حسين ، مؤسسة هنداوي ، القاهرة ، 2014.

3-منهج النقد الادبي ، انريك اندرسون امبرت ، ترجمة الظاهر احمد مكي ، دار العلم العربي ، القاهرة ، 1996.

4-اشكاليات القراءة واليات التأويل ، نصر حامد ابو زيد ،المركز الثقافي العربي لبنان - المغرب ، الطبعة الثالثة ، 1994. 5-المفكرة النقدية ، بشرى موسى صالح ، الطبعة الاولى ، بغداد 2008. 
6-القراءة والتجريب ، حول التجريب في الخطاب الروائي الجديد في المغرب ، د. سعيد يقطين ، رؤية للنشر والتوزيع 2014، الطبعة الاولى .

7-البدايات - القصد والمنهج ، ادوارد سعيد ، نيويورك basic book.

8-جماليات التحليل الثقافي _ الثعر الجاهلي نموذجا ، يونس عليمات ، المؤسسة العربية للدراسات والنشر ، بيروت 2004. 9-نقد النقد ، رواية تعلم ، تزفتيان تودوروف ، ترجمة سامي سويدان ، مركز الانماء القومي ، بيروت1986.

10-علم السرد ، الثكل والوظيفة في السرد ، تأليف جيرالد برنس ، ترجمة الدكتورباسم صالح ، دار الكتب العلمية ، بيروت لبنان 2012.

11-كث وطن ، شهيد ،دار سطور للنشر والتوزيع ، بغداد شارع المتنبي ، الطبعة الثالثة ، 2016 12-بوهيميا الخراب ، صلاح صلاح ، بيروت لبنان ، الطبعة الثالثة ،2009. 13-قاريء الطين ، حسن فالح ، دار سطور للنشر والتوزيع ، بعداد شارع المتببي ، الطبعة الاولى 2016. 14-دفاتر مفككة الاوراق ، فوزي الهنداوي ، دار الجواهري للنشر والتوزيع ،الطبعة الاولى 2016 15-قاموس مصطلحات الانثروبولوجيا والفلكلور ، ايكة هو للتكراس ، ترجمة محمد الجوهري وحسن الثافي ، دار المعارف مصر 1972. 16-النقد الادبي ، ولماذا ،عبد النبي اصطيف ، مجلة فصول ، مصدر سابق . 17-مدخل لسوسيولوجيا القراءة ، جاك لينهاردن ، ترجمة احمد المديني مجلة الفكر العربي المعاصر ، عدد 13 ، سنة

18-الثقافة والتقسير الانثروبولوجي ، ترجمة تراجي فتحي ، المجلس الوطني للثقافة والفنون والاداب ، سلسلة عالم المعرفة ، الكويت 2008.

19-تاريخ النقد الادبي عند العرب ، احسان عباس ، دار الثقافة بيروت ، ط4 ، 1983. 


\section{مجلة لارك للفلسفة واللسدانيات و العلوم الاجتماعية المجلد (2) العدد (41) السذة (2021) بحوث اللغة العربية}

20-النقد الثقافي ، تمهيد مبئي للمفاهيم الرئيسية ، ترجمة وفاء ابراهيم ، رمضان بسطاويسي ، المجلس الاعلى للثقافة ،

21- جماليات التحليل الثقافي -الثعر الجاهلي نموذجا ، يونس عليمات ، المؤسسة العربية للاراسات والنشر ، بيروت ،

مجلة فصول ، مجلة النقد الادبي ، فصلية محكمة ، المجلد 3/25، العدد 99،ربيع 2019.

1-Introduction to Ibn Khaldun, Abd al-Rahman Ibn Khaldun, Dar Al-Qalam, Beirut, Edition 4,1981 .

2- The Hadith on Wednesday, Taha Hussein, Hindawi Foundation, Cairo, 2014.

3- The Literary Criticism Methodology, by Enrique Anderson-Imbert, translated by Al-Zahir Ahmad Makki, Dar Al-Alam Al-Arabi, Cairo, 1996.

4- Problems of Reading and the Mechanisms of Interpretation, Nasr Hamid Abu Zeid, Arab Cultural Center, Lebanon - Morocco, Third Edition, 1994.

5 - The Critical Notebook, Bushra Musa Saleh, First Edition, Baghdad 2008.

6- Reading and experimenting, on experimentation in the new narrative discourse in Morocco,

Dr. Saeed Yoktin, Vision for Publishing and Distribution 2014, First Edition.

7- The Beginnings - Intent and Method, Edward Said, New York, Basic Book.

8- Fosoul Magazine, Literary Criticism Magazine, Court Quarterly, Volume 25/3, Issue 99, Spring 2019. 
9- Aesthetics of Cultural Analysis _ Pre-Islamic Poetry as a Model, Youssef Alabat, Arab Foundation for Studies and Publishing, Beirut, 2004.

10- Criticism of criticism, a learning novel, Tzftian Todorov, translated by Sami Sweidan, National Development Center, Beirut 1986.

" 11- The science of narration, form and function in narration, written by Gerald Prince, translated by Dr.Basem Saleh, Dar Al-Kotob Al-Alami, Beirut - Lebanon, 2012.

12- Kesh Watan, Shahid, Sutour House for Publishing and Distribution, Baghdad Al-Mutanabi Street, Third Edition, 2016

13- Bohemia Desolation, Salah Salah, Beirut Lebanon, Third Edition, 2009.

14- Al-Tud Reader, Hassan Faleh, Sutour House for Publishing and Distribution, by AlMutanabi Street Counter, First Edition 2016.

15-Dismantled Notebooks, Fawzi Al-Hindawi, Dar Al-Jawahiri for Publishing and Distribution, First Edition 2016

16- Dictionary of Anthropology and Folklore Terms, Ikeh is for Consecration, translated by Muhammad al-Gohary and Hassan al-Shafi, Dar al-Ma'arif, Egypt, 1972.

17- Literary Criticism and Why, Abd al-Nabi Istif, Fusul Magazine, previous source.

18- An Introduction to the Sociology of Reading, by Jacques Leinhardn, translated by Ahmad Al-Madani, Journal of Contemporary Arab Thought, No. 13, year 1981.

19- Culture and Anthropological Interpretation, translated by Taraji Fathy, National Council for Culture, Arts and Literature, World of Knowledge Series, Kuwait 2008. 
مجلة لارك لدفلسفة و اللسداذيات و العلوم الاجتماعية المجلد (2) العدد (41) السذة (2021) بحوث اللغة العربية 20- History of literary criticism among the Arabs, Inssan Abbas, Dar Al Thaqafa, Beirut, 4th Edition, 1983.

21-Cultural Criticism, A Preliminary Introduction to the Key Concepts, translated by Wafa Ibrahim, Ramadan Bastawissi, The Supreme Council for Culture, 2003

22- Multiple Lights and One World - Communication and Society, National Publishing Company publications, Algeria.

23-Aesthetics of Cultural Analysis - Pre-Islamic Poetry as a Model, Yunus Alimat, Arab Foundation for Studies and Publishing, Beirut, 2004. 